\section{OP0148 IMPACT OF A CARDIOVASCULAR EVENT ON DMARD TREATMENT AMONG PATIENTS WITH RHEUMATOID ARTHRITIS, PSORIATIC ARTHRITIS, OR PSORIASIS}

J.A. Sparks ${ }^{1}$, T. Lesperance ${ }^{2}$, N.A. Accortt ${ }^{2}$, D.H. Solomon ${ }^{1} .{ }^{1}$ Harvard Medical School, Brigham and Women's Hospital, Boston; ${ }^{2}$ Amgen, Inc, Thousand Oaks, United States

Background: Chronic inflammatory diseases such as rheumatoid arthritis (RA), psoriatic arthritis ( $\mathrm{PsA}$ ), and psoriasis ( $\mathrm{PsO}$ ) increase the risk of cardiovascular (CV) disease. However, a gap in knowledge exists regarding detailed information on changes in immunosuppressive (DMARD) treatments after a CV event. Objectives: We describe treatment patterns among patients with RA, PsA, or PsO who were being treated with DMARDs prior to a CV event.

Methods: Patients with RA, PsA, or PsO, who experienced a CV event (acute myocardial infarction, stroke, or cardiac revascularization) between 1/1/2006 and $6 / 30 / 2015$ were identified in an administrative claims database. Index date was defined as the hospital discharge date for the first CV event during the study period. Patients were required to: be continuously enrolled for 12 months prior to index date; have $\geq 1 \mathrm{TNFi}$ claim, or a conventional synthetic (cs)DMARD claim, or another biologic DMARD claim within 6 months prior to the index date; and have $>30$ days of follow-up after index date. Treatment patterns were assessed after index date and patients were classified as remaining on ("persistent"), switching, or discontinuing pre-index DMARD medication.

Results: We identified 9,529 patients with RA, PsA, or PsO; prior to the index date, $3,274(34.4 \%)$ patients were on TNFi, $5,177(54.3 \%)$ were on only csDMARDs as monotherapy or combination, and 1,078 (11.3\%) were on non-TNFi biologics. Patients on csDMARDs at index date were older (69.4 yrs) than those on TNFi (64.1 yrs) or other biologic DMARDs (66.0 yrs). Approximately $73 \%$ of patients were persistent on their pre-CV event treatment, with higher persistence among csDMARD alone $(76.0 \%)$ and TNFi + csDMARD combo $(76.7 \%)$ groups and lower in the non-TNFi biologic + csDMARD combo group $(60.8 \%$, Table 1$)$. Across all treatment groups, $95 \%$ of persistent patients resumed treatment within 90 days after the index $\mathrm{CV}$ event. Combination therapy users switched their pre-CV event treatment more than monotherapy users, with non-TNFi biologic users more likely to discontinue all therapy after index CV event. Patients that discontinued all therapy after an index CV event tended to be slightly older females (68.7 yrs vs $67.1 \mathrm{yrs})$, with a history of PsO (24.4\% vs $16.1 \%)$, and stroke as index event $(49.3 \%$ vs $41.3 \%)$ compared to those that continued therapy.

Conclusions: In a large US database reflective of typical clinical practice, nearly one-quarter of patients with RA, PsA, or PsO discontinued or switched the pre-event DMARD treatment after a CV event. Further research is needed on whether these DMARD treatment patterns after initial CV event affect risk of repeat CV event.

Disclosure of Interest: J. Sparks Grant/research support from: Amgen, Inc., T. Lesperance Consultant for: Amgen, Inc., N. Accortt Shareholder of: Amgen, Inc., Employee of: Amgen, Inc., D. Solomon Grant/research support from: Amgen, Inc. DOI: 10.1136/annrheumdis-2017-eular.2324

\section{OP0149 MORTALITY IN NEW-ONSET RHEUMATOID ARTHRITIS - HAS MODERN RHEUMATOLOGY HAD AN IMPACT?}

M. Holmqvist ${ }^{1}$, L. Ljung ${ }^{2}$, J. Askling ${ }^{1} .{ }^{1}$ Karolinska Institutet, Stockholm; ${ }^{2}$ Umeå University, Umeå, Sweden

Background: A wealth of studies have linked rheumatoid arthritis (RA) with an excess mortality compared to the general population. With increasingly effective anti-rheumatic treatment strategies there is, however, considerable uncertainty whether this mortality gap still exists and, if so, how soon after RA onset it occurs. Objectives: To assess the mortality in RA compared to the general population with specific focus on when during the course of the disease the risk is increased and if it also applies to patients diagnosed in recent years.

Methods: We performed a population-based cohort study of 17,512 patients with new-onset RA (identified from the Swedish Rheumatology Quality Register) 1997 through 2015 , and 78,847 individually matched general population comparator subjects. We followed all individuals using nationwide census registers with full coverage to identify all deaths through 2015 . We calculated mortality rates with $95 \%$ confidence intervals $(\mathrm{Cl})$ and compared the mortality in RA to that in the general population using Cox proportional hazards models adjusted for age, sex, year of diagnosis, and residential area.

Results: During a mean follow-up from RA diagnosis of 7 years, 2,386 RA patients and 9,850 population comparator subjects died (crude incidence: 19 per 1000 in RA and 18 per 1000 in the general population), with only a marginal decline (in the RA and in the general population cohort) during the study period. The overall HR was $0.99(0.95-1.04)$, but whereas there was no increase in mortality during the first five years after RA diagnosis; the $H R \geq 10$ years after RA onset was $40 \%$ increased. The overall pattern of HRs was similar for patients diagnosed 1997-2001, 2002-2006, and 2007-2011 (table).

Conclusions: The five-year mortality in RA is not increased, neither for patients diagnosed in the past nor for those diagnosed during the most recent five years. By contrast, at least in the most recent inception cohort for which ten-year mortality currently can be calculated (those diagnosed up to 2006), RA is still associated with an increased risk of death.

Disclosure of Interest: None declared

DOI: 10.1136/annrheumdis-2017-eular.2580

\section{OP0150 PARADOXICAL EFFECT OF BIOLOGICAL DMARDS IN RHEUMATOID ARTHRITIS PATIENTS WITH OVERWEIGHT AND OBESITY: LESS OFTEN CLINICAL REMISSION, BUT ALSO LESS RADIOLOGICAL DAMAGE}

A.V. de Jonge ${ }^{1}$, M.M. ter Wee ${ }^{1,2}$, I.E. Bultink ${ }^{1}$, W.F. Lems ${ }^{1}{ }^{1}{ }^{1}$ U University medical centre, Amsterdam Rheumatology and immunology centre; ${ }^{2}$ Epidemiology and Biostatistics, VU University medical center, Amsterdam, Netherlands

Background: The relationship between treatment with biological diseasemodifying antirheumatic drugs (DMARDs), including TNF-blocking agents, and disease activity in overweight and obese rheumatoid arthritis (RA) patients has not been clarified yet.

Objectives: The aim of this review is to assess the effect of overweight/obesity on the therapeutic efficacy of these drugs. Secondly, we aimed to assess the influence of overweight/obesity on the occurrence of joint destruction.

Methods: A systematic review of all articles published on these subjects using PubMed and EMBASE was executed. For the first research question, studies were eligible when focused on the clinical efficacy of biological DMARDs only in overweight/obese RA patients versus patients with normal body weight. For the second research question studies were eligible when questioning the relation between overweight and joint destruction in patients with RA. Overweight and obesity were defined according to the following body mass index (BMI) categories; $\mathrm{BMI}<20 \mathrm{~kg} / \mathrm{m}^{2}$ for underweight, BMl $20-25 \mathrm{~kg} / \mathrm{m}^{2}$ for normal weight, BMI 25-30 $\mathrm{kg} / \mathrm{m}^{2}$ and $\mathrm{BMI}>30 \mathrm{~kg} / \mathrm{m}^{2}$ for overweight.

Results: A total of 6782 articles were found, of which 12 were eligible for this review. A total of 3647 RA patients were treated with adalimumab, etanercept, infliximab, golimumab, or certolizumab pegol, or TNF blockers, rituximab, or tocilizumab.

Ten studies used disease activity as outcome. In general, these studies showed that higher BMI is associated with poor response, based on either outcome or percentages on remission or improvement defined according to EULAR guidelines. In addition, four studies showed that higher BMI is also associated with higher Health Assessment Questionnaire (HAQ)-scores.

Two articles (of which one article described the results of studies in two different RA cohorts) focused on the association between BMI and joint destruction. These articles showed that higher BMI values were associated with lower odds for having joint destruction. One study also showed that having a BMI $<20 \mathrm{~kg} / \mathrm{m}^{2}$ was associated with a higher odds ratio $(\mathrm{OR}=4.12)$ for joint destruction.

Conclusions: Higher BMI levels in RA patients treated with biological DMARDs

Abstract OP0148 - Table 1. DMARD treatment patterns for RA, PsA, or PsO patients following an initial CV event $(\mathrm{N}=9,529)$

\begin{tabular}{|c|c|c|c|c|}
\hline Treatment prior to $\mathrm{CV}$ event & $\mathrm{N}$ & DMARD treatment persisted, $\mathrm{n}(\%)$ & DMARD treatment switched, $\mathrm{n}(\%)$ & All DMARDs treatment discontinued, $\mathrm{n}(\%)$ \\
\hline Entire study sample & 9,529 & $6,985(73.3 \%)$ & $1,498(15.7 \%)$ & $1,046(11.0 \%)$ \\
\hline TNFi monotherapy & 1,760 & $1,323(75.2 \%)$ & $232(13.2 \%)$ & $205(11.6 \%)$ \\
\hline TNFi + csDMARD combination therapy & 1,514 & $1,160(76.7 \%)$ & $299(19.7 \%)$ & $55(3.6 \%)$ \\
\hline csDMARD monotherapy & 4,369 & $3,320(76.0 \%)$ & $528(12.1 \%)$ & $521(11.9 \%)$ \\
\hline$\geq 2$ csDMARDs combination therapy & 808 & $518(64.1 \%)$ & $248(30.7 \%)$ & $42(5.2 \%)$ \\
\hline Non-TNFi biologic monotherapy & 718 & $445(62.0 \%)$ & $63(8.8 \%)$ & $210(29.2 \%)$ \\
\hline Non-TNFi biologic + csDMARD combination therapy & 360 & $219(60.8 \%)$ & $128(35.6 \%)$ & $13(3.6 \%)$ \\
\hline
\end{tabular}

Abstract OP0149 - Table 1. Hazard ratio (HR) and 95\% confidence intervals (Cl) adjusted for sex, residential area, year of diagnosis, and age. Overall and stratified by calendar period of RA diagnosis and time since RA diagnosis

\begin{tabular}{|c|c|c|c|c|c|}
\hline \multirow[b]{2}{*}{ Calendar period of RA diagnosis } & \multicolumn{5}{|c|}{ RA duration categories $\mathrm{HR}(95 \% \mathrm{Cl})$} \\
\hline & Total follow-up & $<1$ year & $1-<5$ years & $5-10$ years & $>10$ years \\
\hline Total study period & $1.01(0.96-1.06)$ & $0.57(0.48-0.67)$ & $0.90(0.83-0.97)$ & $1.11(1.02-1.20)$ & $1.43(1.28-1.59)$ \\
\hline $1997-2001$ & $1.09(1.01-1.18)$ & $0.58(0.39-0.87)$ & $0.81(0.68-0.96)$ & $1.06(0.92-1.21)$ & $1.41(1.25-1.60)$ \\
\hline $2002-2006$ & $1.02(0.94-1.10)$ & $0.37(0.24-0.57)$ & $0.88(0.76-1.01)$ & $1.15(1.03-1.30)$ & $1.48(1.20-1.82)$ \\
\hline 2007-2011 & $0.95(0.86-1.05)$ & $0.65(0.49-0.87)$ & $0.98(0.86-1.11)$ & $1.09(0.90-1.31)$ & - \\
\hline 2012-2015 & $0.77(0.63-0.95)$ & $0.63(0.46-0.88)$ & $0.87(0.67-1.12)$ & - & - \\
\hline
\end{tabular}


are associated with reduced therapeutic efficacy as compared to RA patients with normal body composition. The unfavourable effect of body weight on disease activity was paralleled by a favourable, protective effect on joint destruction. This uncoupling is not fully elucidated yet, and should be further investigated.

Disclosure of Interest: None declared

DOI: 10.1136/annrheumdis-2017-eular.4694

\section{OP0151 DOCOSAHEXAENOIC ACID TREATMENT OF RHEUMATOID ARTHRITIS: A RANDOMIZED, DOUBLE-BLIND, PLACEBO-CONTROLLED, CROSS-OVER STUDY}

T. Neumann ${ }^{1,2}$, C. Dawczynski ${ }^{3,4}$, M. Dittrich ${ }^{5}$, K. Goetze ${ }^{6}$, A. Welzel ${ }^{1}$, P. Oelzner ${ }^{1}$, S. Voelker ${ }^{7}$, A.M. Schaible ${ }^{7}$, F. Troisi ${ }^{7}$, L. Thomas ${ }^{7}$, S. Pace ${ }^{7}$ A. Koeberle ${ }^{7}$, O. Werz ${ }^{7}$, P. Schlattmann ${ }^{8}$, S. Lorkowski ${ }^{3,4}$, G. Jahreis ${ }^{3,4}$.

${ }^{1}$ Department of Internal Medicine III, Jena University Hospital, Jena, Germany;

${ }^{2}$ Department of Rheumatology, Kantonsspital St. Gallen, St. Gallen, Switzerland;

${ }^{3}$ Competence Cluster for Nutrition and Cardiovascular Health (nutriCARD);

${ }^{4}$ Department of Nutritional Biochemistry and Physiology, Institute of Nutrition;

${ }^{5}$ Department of Nutritional Physiology, Institute of Nutrition, Friedrich Schiller

University of Jena; ${ }^{6}$ Department of Rheumatology, Jena University Hospital;

${ }^{7}$ Department of Pharmaceutical/Medicinal Chemistry; ${ }^{8}$ Department of Medical

Statistics, Informatics and Documentation, Friedrich Schiller University of Jena,

Jena, Germany

Background: Epidemiological studies and clinical investigations indicate a beneficial impact of long-chain n-3 polyunsaturated fatty acids ( $n-3$ PUFA) on the inflammatory activity of rheumatoid arthritis (RA). However, the knowledge about the physiological effects of the individual compounds eicosapentaenoic acid (EPA) and docosahexaenoic acid (DHA) is limited.

Objectives: In our pilot study presented here, we investigated the clinical benefit of daily intake of foods enriched with microalgae oil as source of DHA in RA patients. In particular, the influence on disease activity and changes in the profile of pro-inflammatory/non-resolving and anti-inflammatory/pro-resolving lipid mediators was examined.

Methods: This is a randomized, double-blind, cross-over study on 38 patients (32 females/ 6 males) with active RA (DAS28 $\geq 2.4$ ). They were allocated to consume foods enriched with microalgae oil from Schizochytrium $s p$. (2.1 g DHA/day) or sunflower oil (placebo) for 10 weeks while maintaining stable immunosuppressive treatment.

Results: Thirty-two patients finished the study but seven patients were excluded from analysis because their DHA increase in erythrocyte lipids (EL) was less than $25 \%$ after 10 weeks of intervention indicating insufficient adherence to the intervention. Supplementation of DHA led to a decline in tender joints (TJ68) $8.4+5.6$ to $6.0+5.0(\mathrm{p}=0.03)$, swollen joints (SJ66) $5.6 \pm 3.5$ to $3.9 \pm 3.5(\mathrm{p}=0.07)$ and DAS28 $4.3 \pm 1.0$ to $3.9 \pm 1.2(p=0.07)$. Joint counts in the placebo arm remained stable (TJ68) $6.7 \pm 4.9$ to $8.8 \pm 8.0 \quad(p=0.12)$, swollen joints (SJ66) $3.5 \pm 3.0$ to 4.2 $\pm 3.7(\mathrm{p}=0.40)$ and DAS28 $4.0 \pm 0.9$ to $4.1 \pm 1.2(\mathrm{p}=0.45)$. Ultrasound score (US-7) remained stable ( $15.1 \pm 9.5$ to $12.4 \pm 7.0 ; p=0.160)$ while it increased in the placebo arm $(11.4 \pm 7.0$ to $14.0 \pm 8.8 ; p=0.03)$.

The amount of $\mathrm{n}-3$ PUFA in erythrocyte increased in supplemented patients ( $7.0 \pm 1.2 \%$ to $10.6 \pm 1.4 \% ; p<0.001)$ and the ratios of aracidonic acid (AA) /EPA (18.5 \pm 6.3 to $12.6 \pm 5.5 ; p<0.001)$ and $A A / D H A(3.8 \pm 1.0$ to $1.8 \pm 0.6 ; p<0.001)$ dropped significantly. Fatty acid distribution in erythrocyte lipids (n-3 PUFA, $A A / E P A$ and $A A / D H A)$ remained stable in the control arm.

Conclusions: The study shows that microalgae DHA supplements can ameliorate disease activity in patients with active RA along with a shift in the balance of AA- and DHA-derived lipid mediators towards an anti-inflammatory/pro-resolving state.

Disclosure of Interest: None declared

DOI: 10.1136/annrheumdis-2017-eular.6759

\section{OP0152 A LONGITUDINAL STUDY OF THE EFFECTS OF DISEASE ACTIVITY ON RENAL FUNCTION IN PATIENTS WITH RHEUMATOID ARTHRITIS UTILIZING LINEAR MIXED EFFECT MODELS - ANSWER COHORT STUDY -}

A. Onishi ${ }^{1}$, K. Akashi ${ }^{1}$, M. Hashimoto ${ }^{2}$, M. Furu ${ }^{2}$, W. Yamamoto ${ }^{2}$, M. Katayama ${ }^{3}$, R. Hara ${ }^{4}$, T. Fujimura ${ }^{4}$, S. Yoshida ${ }^{5}$, K. Nagai ${ }^{5}$, T. Hirano ${ }^{6}$, K. Ebina ${ }^{7}$, Y. Son ${ }^{8}$, H. Amuro ${ }^{8}$, H. Yamada ${ }^{1}$, Y. Ichise ${ }^{1}$, D. Waki ${ }^{1}$, I. Naka ${ }^{1}$, K. Tsuda ${ }^{1}$, T. Okano ${ }^{1}$, S. Takahashi ${ }^{1}$, S. Sendo ${ }^{1}$, Y. Ueda ${ }^{1}$, Y. Kogata ${ }^{1}$, J. Saegusa ${ }^{1}$, A. Morinobu ${ }^{1} .{ }^{1}$ Department of Rheumatology, Kobe University Hospital, Kobe; ${ }^{2}$ Department of the Control for Rheumatic diseases, Graduate School of Medicine, Kyoto University, Kyoto; ${ }^{3}$ Department of Rheumatology and Clinical Immunology, Osaka Red Cross Hospital, Osaka; ${ }^{4}$ The Center for Rheumatic Diseases, Nara Medical University, Nara; ${ }^{5}$ Department of Internal Medicine (I), Osaka Medical College; ${ }^{6}$ Department of Respiratory Medicine, Allergy and Rheumatic Disease, Graduate School of Medicine, Osaka University; ${ }^{7}$ Department of Orthopaedic Surgery, Osaka University, Graduate School of Medicine: ${ }^{8}$ First Department of Internal Medicine, Kansai Medical University, Osaka, Japan

Background: Patients with RA have higher risk of developing chronic kidney disease compared to the general population. Although some causes of renal impairment in RA include nephrotoxic medications, amyloidosis, and glomerulonephritis, specific causes are not determined among most patients with RA. While experimental researches showed inflammatory process per se might also contribute to renal dysfunction, the results of previous clinical studies that assessed the effects of disease activity or C-reactive protein on renal function were inconsistent. This inconsistency might be attributable to their cross-sectional design, small sample size, and assessment of association between only baseline characteristics and renal outcomes without consideration for the fact that disease activity and medications had changed over time.

Objectives: To identify the effects of disease activity on renal function in RA in a multi-center cohort study.

Methods: RA patients with a sampling interval of less than 150 days were enrolled because wide sampling intervals could not take into consideration changes in disease activity and medications during their follow-up. An estimated glomerular filtration rate (eGFR) was calculated using an equation approved by the Japanese Society of Nephrology and used as an outcome variable. Linear mixed effect models were used to evaluate the renal trajectories of patients. Time from baseline (months), disease activity, and their interaction were included as fixed effects while participant identification number and time from baseline were included as random factors. Age, sex, disease duration, RF, ACPA, NSAIDs, and DMARDs that were known as a cause of renal impairment, such as tacrolimus, iguratimod, and tofacitinib, were included as covariates.

Results: A total of 25661 samples (mean sampling interval: 2.0 months) from 2104 patients was included. Patients with lower DAS28-CRP had worse renal function at inclusion, but a significantly better longitudinal trajectory on eGFR $\left(0.0079 \mathrm{ml} / \mathrm{min} / 1.73 \mathrm{~m}^{2}\right.$ per month, $\left.\mathrm{P}=0.025\right)$. Although all $\mathrm{RA}$ patients had naturally progressive renal impairment as they got older, patients who achieved remission or low disease activity had slower renal impairment rate of -0.068 $\mathrm{ml} / \mathrm{min} / 1.73 \mathrm{~m}^{2}$ per month compared to patients with moderate or high disease activity $\left(-0.084 \mathrm{ml} / \mathrm{min} / 1.73 \mathrm{~m}^{2}\right.$ per month; $\left.\mathrm{P}=0.037\right)$. These results were also similar using SDAI or CDAI.

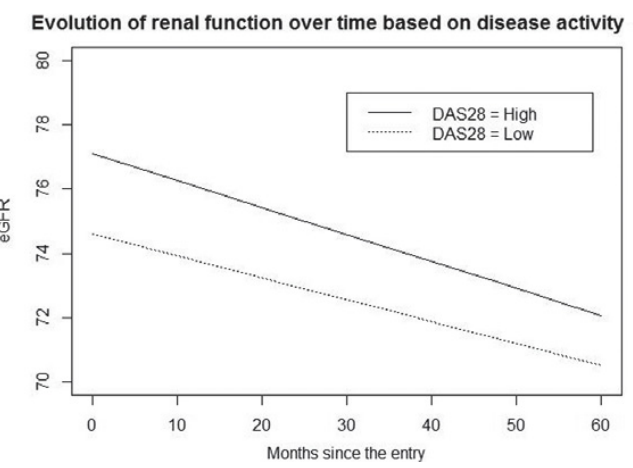

Conclusions: Lower disease activity results in slower renal impairment. Because the effects of disease activity on renal function are mild, additional measures to protect renal function, such as avoiding nephrotoxic medications and treating cardiovascular risk factors are important.

Disclosure of Interest: None declared DOI: 10.1136/annrheumdis-2017-eular.4544

\section{OP0153 RECURRENT INFECTIONS IN RHEUMATOID ARTHRITIS PATIENTS, RESULTS FROM THE BSRBR}

S. Subesinghe ${ }^{1}$, A.I. Rutherford ${ }^{1}$, R. Byng-Maddick ${ }^{2}$, J.B. Galloway ${ }^{1}$. ${ }^{1}$ Rheumatology, King's College London; ${ }^{2}$ Homerton Hospital, London, United Kingdom

Background: Rheumatoid arthritis (RA) patients have an increased susceptibility to infection.

Objectives: 1.To establish the rate of recurrent infection in RA patients recruited to the British Society of Rheumatology Biologics Registry Rheumatoid Arthritis (BSRBR-RA).2.To establish whether the organ class of index infection predicted future serious infection.

Methods: The BSRBR-RA is a prospective observational cohort, previously described. Patients with at least one episode of serious infection requiring hospitalisation were included if they occurred whilst on anti-rheumatic drug therapy or within 5 drug half-lives of stopping. Infections were coded by MedDRA classification in to 7 categories. Infections occurring over 14 days after the first index infection were considered as new events. Event rates were calculated and compared using a Cox proportional hazards model with adjustments made for age, gender, disease duration, baseline DAS28 score, smoking status and seropositivity.

Results: See Table 1.

In total, 21,943 subjects with 115,423 patient-years follow up were studied, 5365 subjects reported at least one serious infection. Comparing organ classes of prior infection at baseline, each group had comparable age, disease duration, baseline DAS28 and HAQ scores. The cohort characteristics are tabulated. The baseline annual rate of first serious infection was $4.6 \%(95 \% \mathrm{Cl} 4.5-4.7)$. Following an 